\title{
Erratum
}

\section{On the existence of a local quasi hidden variable (LqHV) model for each $N$-qudit state and the maximal quantum violation of Bell inequalities}

[Int. J. Quant. Phys., Vol. 14, No. 4 (2016) 1640010 (15 pages)]

Elena R. Loubenets

National Research University Higher School of Economics, Moscow 110000, Russia

Published 26 December 2016

In the published version of this article, in the sentence on page $1640010-2$, line 16 , the word "no" is missed. The correct form of this sentence should be: "Nowadays, there is still no a unique conceptual view ${ }^{\mathrm{c}}$ on Bell's concept of quantum nonlocality". 